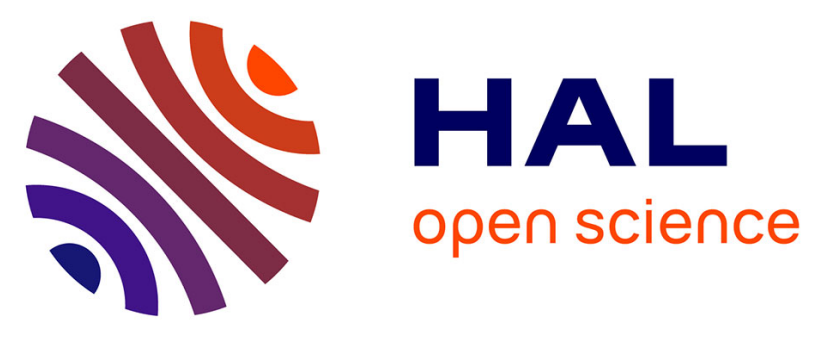

\title{
Comparison between numerical and experimental results on thermoconvective instabilities of a high-Prandtl-number liquid
}

Emilien Sauvage, Laetitia Jacoutot, Yves Fautrelle, Annie Gagnoud, Laure Blumenfeld, Eric Favre, François Daviaud

\section{To cite this version:}

Emilien Sauvage, Laetitia Jacoutot, Yves Fautrelle, Annie Gagnoud, Laure Blumenfeld, et al.. Comparison between numerical and experimental results on thermoconvective instabilities of a highPrandtl-number liquid. Physical Review E: Statistical, Nonlinear, and Soft Matter Physics, 2007, 76 (6), pp.066307. 10.1103/PhysRevE.76.066307 . cea-01373399

HAL Id: cea-01373399 https://hal-cea.archives-ouvertes.fr/cea-01373399

Submitted on 28 Sep 2016

HAL is a multi-disciplinary open access archive for the deposit and dissemination of scientific research documents, whether they are published or not. The documents may come from teaching and research institutions in France or abroad, or from public or private research centers.
L'archive ouverte pluridisciplinaire HAL, est destinée au dépôt et à la diffusion de documents scientifiques de niveau recherche, publiés ou non, émanant des établissements d'enseignement et de recherche français ou étrangers, des laboratoires publics ou privés. 


\title{
Comparison between numerical and experimental results on thermoconvective instabilities of a high-Prandtl-number liquid
}

\author{
Emilien Sauvage, ${ }^{*}$ Laetitia Jacoutot, ${ }^{\dagger}$ Yves Fautrelle, and Annie Gagnoud \\ SIMAP Groupe EPM, ENSEEG, BP 75, 38402 Saint Martin d'Hères, France \\ Laure Blumenfeld and Eric Favre \\ SFME/LTMF, CEA Saclay, F-91191 Gif-sur-Yvette Cedex, France \\ François Daviaud \\ DSM/DRECAM/SPEC, CEA Saclay, F-91191 Gif-sur-Yvette Cedex, France
}

(Received 23 April 2007; published 10 December 2007)

\begin{abstract}
The flow structuration of silicon oil (Prandtl number of 10.3) in a open cylindrical pool heated from the center of the surface is investigated numerically. Our purpose is to perform the numerical simulation of experimental results obtained by Favre et al. [Phys. Fluids 9, 1473 (1997)] who observed transitions between steady and axisymmetric flows at sufficiently low values of the Marangoni number (Ma) and various types of instability depending on the height of the fluid. The hydrothermal wave regime has been obtained at critical values of Ma which depend on the Bond number and on the aspect ratio. The numerical results are in good agreement with the experimental ones.
\end{abstract}

DOI: 10.1103/PhysRevE.76.066307

PACS number(s): 47.20.-k, 47.35.-i

\section{INTRODUCTION}

When a liquid free surface is submitted to a horizontal thermal gradient, there appears a surface stress which drives a flow along the interface. The latter phenomenon called the thermocapillary effect, also called the Marangoni effect, comes from the existence of variations of the surface tension along the interface correlated to the temperature variations $\Delta T$. Generally, thermocapillary effects are coupled with natural convection. Since buoyancy is a body force, its importance depends on the volume of the pool in comparison with its free surface.

The thermocapillary effect is found in various industrial processes. For example, in welding devices by means of electron/laser beam or arc, the molten pool is heated by its free surface, and there exist very strong temperature gradients along the surface [1-3]. The Marangoni effect is usually dominant, and significant fluid flows are observed. Similar situations are encountered in evaporating devices which use concentrated heat sources (laser or electron beam) for the coating of substrates by vapor deposition.

In crystal growth, thermocapillary effects are also present, although less intense [4]. Nevertheless, Marangoni convection is responsible for various defects, e.g., striations of the solid crystal, which result from the flow instabilities of the thermocapillary type.

The aim of the present paper is to analyze, thanks to numerical calculations, the various types of flow configurations obtained experimentally by Favre [2,3]. First the different types of flow and instabilities put forth in the literature will be reviewed hereafter in the present section. Then in Sec. II the formulation of the problem is provided. In Sec. III the

\footnotetext{
*emilien.sauvage@hmg.inpg.fr

${ }^{\dagger}$ Also at Center for Turbulence Research, Stanford, CA.
}

various types of computed flow patterns and instabilities are compared to the experimental data.

Basic flow. Contrary to the Bénard type flow (vertical thermal gradient only) for which a threshold of temperature exists, in this configuration as soon as $\Delta T \neq 0$, the fluid moves. For example, if we consider the simplest case corresponding to a fluid cavity submitted to a horizontal temperature difference $\Delta T$ applied on each lateral side, the flow near the equilibrium state, i.e., small $\Delta T$, is bidimensional and stationary. This flow is named "basic flow." Hydrodynamic theory for the thermocapillary effect is quite old since the basic flow in a rectangular cavity with no gravity was solved by Birikh [5] in 1962 and the first linear stability study was made in 1983 by Smith and Davis [6]. Laure et al. [7] and Mercier and Normand [8] have extended this solution to a flow with thermogravity effect and other boundary conditions. Finally, Garnier [9] proposed an analytical solution to the basic flow in a cylindrical cavity based on a method introduced by Laure et al. [7]. This solution will be extended to the specific case studied experimentally by Favre [2] experiments in which the bottom boundary is cooled.

Instabilities. When the imposed temperature difference $\Delta T$ crosses a certain threshold, the basic flow is destabilized. Instabilities have various forms and characteristics depending on many parameters of the problem such as the Prandtl number, Bond number, aspect ratio, Biot number, and the type of boundary conditions. The most studied case in the literature is a rectangular cavity heated and cooled on both sides by a vertical boundary, the free surface and the bottom being insulating or conducting. In this configuration the thermal gradient is horizontal, which simplifies the analytical solution. Annular geometry is more and more studied because of two reasons. First, in this particular case, the absence of walls in the orthoradial direction (i.e., the direction perpendicular to the thermal gradient) avoids experimentally wave reflection on the side walls. Second, this annular ge- 
ometry is closer to the industrial process of the Czochralski crystal growth method of electron beam and laser surface heating. Very few studies $[10,11]$ have considered a heating configuration localized on the surface, as used in the Favre [2] study. Moreover, the condition of a cooled bottom boundary is also rarely used [12]. The strong variation of the stability criteria with the numerous problem parameters makes the comparison between results of different studies very hard to achieve. However, the instabilities can be sorted out in three groups.

The first type of instability in a similar configuration is the function of the aspect ratio of the fluid layer. For a small Bond number, this instability is composed of corotative stationary or time dependent rolls with a two-dimensional longitudinal axis. Laure et al. [7] give critical values associated to their apparition in a long rectangular cavity with a low Prandtl number fluid. Mercier and Normand [13] have shown that rolls appear near the cold wall for fluid with a low Prandtl number $(\operatorname{Pr}<0.01)$, near the hot wall for fluid with a high Prandtl number $(\operatorname{Pr}>4)$, and near both sides for fluid with an intermediate Prandtl number. These results have been checked in a great number of two-dimensional numerical publications [14-18]. The influence upon the flow structure of parameters as the aspect ratio, the Bond number, and several boundary condition types has been also studied. Some numerical studies [14-16] report the presence of temporal oscillations due to the destabilization of the first corotative roll. Experimentally this instability is well observed for various configuration $[5,12,15,19]$.

The second type of instability consists of stationary rolls with an axis collinear to the thermal gradient direction. This instability has been reported experimentally by Daviaud and Vince [20] in a rectangular cavity and in an annular cavity by Favre [3] for a relatively high height of fluid $(h>3 \mathrm{~mm})$. Li et al. [11] found numerically longitudinal rolls in an annular cavity while Mercier and Normand [8], from an analytical point of view, have predicted this instability for a fluid of Prandtl number $\operatorname{Pr}=7$ in a cavity with conducting thermal boundary conditions at the bottom and introducing a Biot number at the surface. This kind of instability has not been studied widely.

The third and most interesting instability are the hydrothermal waves. Smith and Davis [6] have studied in 1983, the linear stability of a return flow without gravity and discovered this new kind of instability. They appear as a propagative ondulatory instability of the basic flow with specific frequency and wave number. The propagation angle of the waves with the thermal gradient direction is a function of the Prandtl number [6]. Numerous experiences observed these hydrothermal waves in rectangular [19] and annular [12] geometry. A summary is presented by Burguete et al. [21]. A stability diagram of the flow is achieved plotting the critical values of imposed thermal difference (or Marangoni number) for which instability occurs against the height of the fluid in the cavity (or Bond number). The critical Marangoni value increases with the height of fluid. If the height of fluid crosses a certain threshold the instability is not hydrothermal waves any longer but an oscillating multicellular flow. In a majority of studies, the hydrothermal waves are the second instability, the first being the corotative instability. A similar

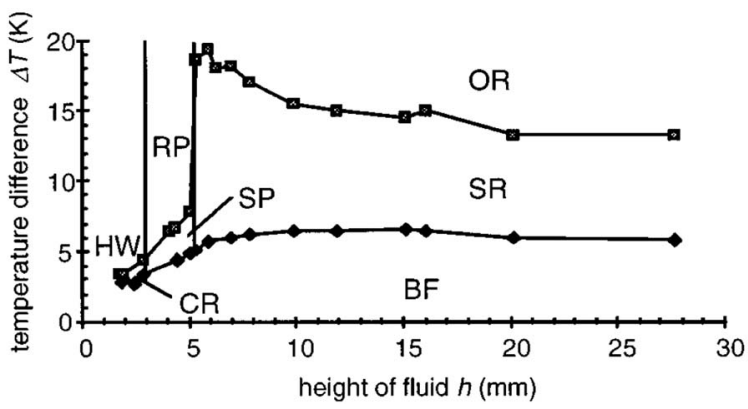

FIG. 1. Diagram of stability due to Favre [2] (see text for details).

stability diagram of the flow has been drawn by Favre [2] which is shown in Fig. 1. Garnier [9] puts forth the assumption that the hydrothermal waves can appear only when $\mathrm{Bd}$ $>1$, i.e., when thermogravitational forces are negligible, a suggestion which is in agreement with the theory of Smith and Davis [6] where only the thermocapillary force was taken into account.

From a numerical point of view, many calculations, especially in two-dimensional geometries, has been achieved (see, for example, Ben Hadid and Roux [17]). The increase of computation capacity leads to the achievement of simulations of the three-dimensional hydrothermal waves. Hoyas and co-workers [22,23] performed a series of threedimensional calculations in an annular geometry with a numerical method based upon the resolution of the set of stability equations with a decomposition of the fields in Chebyshev polynoms. They found, for a fluid with an infinite Prandtl number, hydrothermal waves propagating near the surface and studied the threshold of appearance depending on the aspect ratio and thermal boundary. Li and co-workers [11,24-26] simulate hydrothermal waves in an annular geometry heated either by the outer wall or the surface, solving directly the Navier-Stokes equations with a volume-of-fluid method $(\operatorname{Pr} \ll 1)$. Specifically, they were able to determine both the threshold of apparition of the waves and to visualize them correctly. In 2006, Shi and Imaishi [27] simulated, with the same methods, a fluid of Prandtl number $\operatorname{Pr}=6.7$. Visualization of the hydrothermal waves computed by Shi and Imaishi [27] is similar to that obtained experimentally by Garnier [9].

Experimental results of Favre [2]. The studied geometry is original because the fluid is heated on the surface in the center of the cavity and cooled both by the bottom and side walls. Favre [2] observed the three different types of instability as we can see on the stability diagram in Fig. 1. Whatever the height of fluid $h$, when $\Delta T$ is increased, a transition occurs between a steady basic flow (BF) and a structured stationary flow. The height of fluid determines the type of visualized structures: corotating rolls (CRs), stationary petals (SPs), or stationary rays (SRs). Three shadowgraphic images explaining the name of stationary instabilities are presented in Fig. 2. When $\Delta T$ is further increased, the flow becomes unsteady and a new type of instability could appear. The stationary patterns (CR,SP, SR) are replaced, respectively, by hydrothermal waves (HWs), radial pulsations (RPs), and os- 


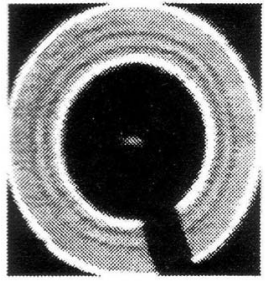

(a)

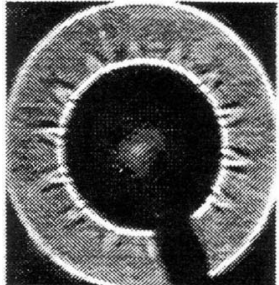

(b)

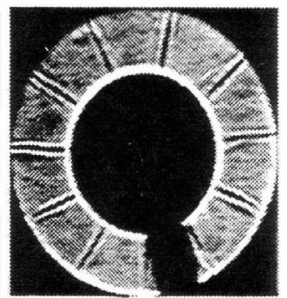

(c)
FIG. 2. Shadow-graphic views of stationary instabilities: (a) corotating rolls (CRs), (b) stationary petals (SPs), and (c) stationary rays (SRs) obtained by Favre [2].

cillating rays (ORs). The structures named "rays" correspond to the second type of instabilities described below, i.e., stationary rolls with an axis collinear to the thermal gradient direction. The "petal" structure has been described in the literature as the superposition of two counter-rotative wave trains but Favre [2] was unable to describe it precisely.

\section{PROBLEM FORMULATION}

\section{A. Physical and mathematical models}

The problem consists of the analysis of a flow in a cylindrical pool with a free surface subjected to a thermal gradient (cf. Fig. 3). The fluid layer of height $h$ is heated at the surface in the center of the cylindrical pool with a heater of radius $R_{1}=15 \mathrm{~mm}$ and cooled at the bottom and side of the pool of external radius $R_{2}=50 \mathrm{~mm}$. The radial length of the pool is $L=R_{2}-R_{1}$, the heater is at the temperature $T_{H}$, while the cooled surfaces are at the temperature $T_{c}$. The typical thermal gradient in the pool is $\Delta T / L=\left(T_{H}-T_{c}\right) / L \geq 0$. We introduce a Biot number $\mathrm{Bi}$ to characterize convective heat transfer on the free surface. The driving forces of the flow are (i) the buoyancy force which tends to drive the hot fluid upward, and (ii) the thermocapillary force which draws the surface fluid from the hot central zone toward the cold boundary. The thermocapillary force is generated by the variation of the surface tension $\sigma$ with the temperature which is well represented by the linear law $\sigma(T)=\sigma_{0}-\gamma\left(T-T_{0}\right)$ where $\gamma=-\partial \sigma / \partial T$ is for common fluids always positive and constant and $\sigma_{0}$ is the value of the surface tension at the temperature $T_{0}$. The physical properties of the silicon oil are summarized in Table I.

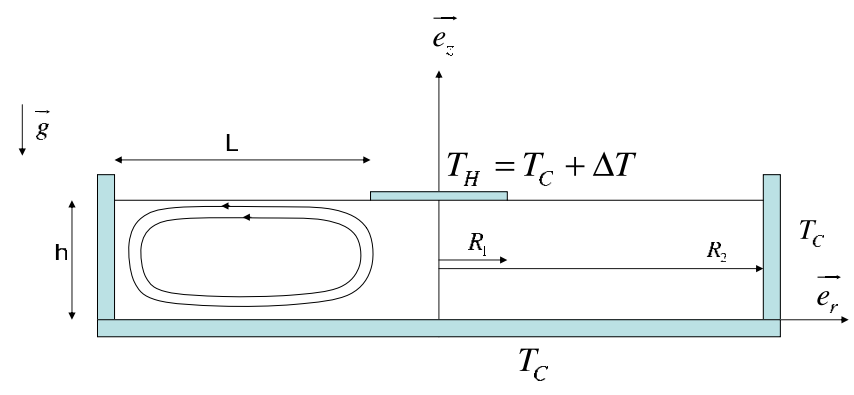

FIG. 3. (Color online) Section of the axisymetric cylindrical geometry and associated notations. Two streamlines of the basic flow are represented symbolically in the left part of the section.
Governing equations. The incompressible governing equations are expressed in a cylindrical reference frame and take into account the buoyancy effect with the Boussinesq approximation,

$$
\begin{gathered}
\rho_{0}\left[\frac{\partial \vec{v}}{\partial t}+(\vec{v} \cdot \vec{\nabla}) \vec{v}\right]=-\vec{\nabla} P+\mu \Delta \vec{v}+\rho_{0} \beta\left(T-T_{0}\right) \vec{g}, \\
\vec{\nabla} \cdot \vec{v}=0, \\
\frac{\partial T}{\partial t}+\vec{v} \cdot \vec{\nabla} T=\kappa \Delta T,
\end{gathered}
$$

where $\vec{v}$ is the velocity vector such as $\vec{v}(r, \theta, z)=u \overrightarrow{e_{r}}+v \overrightarrow{e_{\theta}}$ $+w \overrightarrow{e_{z}}, \beta=\frac{1}{\rho_{0}} \frac{\partial \rho}{\partial T}$ is the thermal expansion coefficient supposed to be constant, and $\rho_{0}$ is the fluid density at the temperature $T_{0}$.

The boundary conditions. At the bottom $z=0$, we use the no-slip condition $\left.\vec{v}\right|_{z=0}=\overrightarrow{0}$, while the temperature is imposed $\left.T\right|_{z=0}=T_{c}$. For $z=h$, the free surface is assumed to be flat and nondeformable, and a no-flux condition is applied, $\vec{v} \cdot \vec{n}=0$. Furthermore, the viscous surface stress is equal to the capillary stress induced by the thermal gradient: $\left.\mu \frac{\partial u}{\partial z}\right|_{z=h}$ $=\left.\gamma \frac{\partial T}{\partial r}\right|_{z=h}$. The thermal condition is $\left.\frac{\partial T}{\partial r}\right|_{z=h}+\operatorname{Bi}\left(T-T_{\infty}\right)=0$, where $\mathrm{Bi}$ is the Biot number and $T_{\infty}$ is the temperature far from the surface being equal to $T_{\text {cond }}$. We introduce the nondimensional parameter $\mu_{B}$ such that $\mu_{B}=\mathrm{Bi} /(1+\mathrm{Bi})$.

This system is characterized by six dimensionless parameters: (i) Prandlt number: $\operatorname{Pr}=\nu / \kappa$; (ii) Rayleigh number $\mathrm{Ra}$ $=g \beta \Delta T h^{4} / \kappa \nu L$; (iii) Marangoni number Ma $=\gamma \Delta T h^{2} / \rho_{0} \kappa \nu L$; (iv) aspect ratios $\varepsilon=h / L$ and (v) $\phi=L / R_{1}$; (vi) Biot number $\mathrm{Bi}=h_{N} h / \lambda$ where $h_{N}$ is the convective heat transfer coefficient at the free surface. Instead of the Rayleigh number we use the dynamic Bond number $\mathrm{Bd}=\mathrm{Ra} / \mathrm{Ma}$. The dynamic Bond number can be written as the square of the ratio between the height of fluid $h$ and the capillary length $h_{e}^{2}$ $=\gamma / \rho_{0} g$.

\section{B. Numerical methods}

The commercial software FLUENT is used for the simulations. The equations are discretized by the finite volume method. The third-order MUSCL scheme (monotone upstream-centered schemes for conservation laws) is used for the convective terms while a second-order centered

TABLE I. Physical properties of the fluid.

\begin{tabular}{ll}
\hline \hline Kinematic viscosity & $\nu=6.5 \times 10^{-7} \mathrm{~m}^{2} \mathrm{~s}^{-1}$ \\
Density & $\rho=760 \mathrm{~kg} \mathrm{~m}^{-3}$ \\
Thermal diffusivity & $\kappa=0.63 \times 10^{-7} \mathrm{~m}^{2} \mathrm{~s}^{-1}$ \\
Thermal conductivity & $\lambda=0.1 \mathrm{~W} \mathrm{~m} \mathrm{~K}^{-1}$ \\
Temperature coefficient & $\gamma=-8 \times 10^{-5} \mathrm{~N} \mathrm{~m}^{-1} \mathrm{~K}^{-1}$ \\
of surface tension & \\
Thermal expansion coefficient & $\beta=1.34 \times 10^{-3} \mathrm{~K}^{-1}$ \\
Prandtl number & $\operatorname{Pr}=10.3$ \\
\hline \hline
\end{tabular}


TABLE II. Grid convergence for the two-dimensional case.

\begin{tabular}{lccccccc}
\hline \hline $\begin{array}{l}\text { Number } \\
\text { of meshes } \\
(r \times z)\end{array}$ & $\begin{array}{c}u(25 ; 14,9) \\
\mathrm{mm} \mathrm{s}^{-1}\end{array}$ & $\begin{array}{c}u(30 ; 14,9) \\
\mathrm{mm} \mathrm{s}^{-1}\end{array}$ & $\begin{array}{c}u(38 ; 14,9) \\
\mathrm{mm} \mathrm{s}^{-1}\end{array}$ & $\begin{array}{c}\text { Pe } \\
\text { Max }\end{array}$ & $\begin{array}{c}\text { mesh } \\
\mathrm{Pe} \leq 1\end{array}$ & $\begin{array}{c}\% \text { mesh } \\
\mathrm{Pe} \geq 10\end{array}$ & Flux (W) \\
\hline 7636 & 5.2 & 2.6 & 1 & 35.8 & & & 0.2197 \\
50700 & 6.6 & 4 & 1.1 & 17.3 & 75 & 1.5 & 0.2237 \\
110000 & 7.3 & 4.8 & 1.1 & 15 & 77 & 0.3 & 0.2258 \\
126000 & 7.6 & 5 & 1.9 & 13 & 78 & 0.2 & 0.2265 \\
\hline \hline
\end{tabular}

scheme is applied to the diffusion terms. The pressure terms are interpolated with the PRESTO! scheme. The SIMPLEC algorithm is used to handle the pressure coupling. The high orders of resolution are useful to simulate correctly the strong coupling between the flow and the temperature field due to the high value of the Prandtl number $(\operatorname{Pr}=10.3)$. Convergence at each time step is considered when residual error of the continuity equation and velocity component became less than $10^{-5}$ and less than $10^{-7}$ for the temperature. $\mathrm{Nu}$ merical simulations were performed on an Intel Bi-pro Xeon 3.80 Ghz, 3.89 Go RAM.

Nonuniform staggered grids are used in this study. The mesh is finer near the free surface and near the cold vertical boundary because of the strong velocity and thermal gradient due to the Marangoni effect and the thermal boundary layer. To assess the effects of numerical diffusion we use the mesh Peclet number, $\mathrm{Pe}_{m}$, which is the ratio of a convection time to a thermal diffusion time at the scale of the mesh, $\mathrm{Pe}_{m}$ $=u_{m} l_{m} / \kappa$ where $u_{m}$ is an average velocity and $l_{m}$ is a mesh characteristic length. A high value of the Peclet number means an important numerical diffusion and gives less accurate results. Several grids were tested. The convergence tests for two-dimensional grids are presented in Table II for the cases $h=14.9 \mathrm{~mm}$ and $2.9 \mathrm{~K}$, while Table III shows the 3Dgrid convergence test for $h=2 \mathrm{~mm}$. The criterion are (i) the value of the heat flow evacuated by the heater, (ii) the radial velocity value at the free surface for three different radii $(25$, 30 , and $38 \mathrm{~mm}$ ), (iii) the maximal and global average value of the Peclet number, and (iv) mesh percentages of the field having a Peclet number, lower than 1 and higher than 10 .

It must be noticed that the values of the radial velocity on the surface are very sensitive to the mesh quality, but the global flow structuration does not really change.

The shadowgraphic method used experimentally by Favre consists of averaging the horizontal thermal gradient along the height of fluid. From a numerical point of view, similar visualizations are obtained by deleting the visual effect of the main radial thermal gradient to emphasize azimuthal variations of the thermal gradient. A first method due to Li et al. [24] consists in computing a fluctuating temperature field $T^{\prime}$ defined as

$$
T^{\prime}(r, z)=T(r, \theta, z)-\frac{1}{2 \pi} \int_{0}^{2 \pi} T(r, \theta, z) d \theta .
$$

The second method consists in post-treating directly the radial or tangential temperature gradient field.

\section{RESULTS AND DISCUSSIONS}

\section{A. Basic flow}

The analytical solution of the thermoconvective basic flow for the cylindrical geometry within the assumption of small aspect ratio is presented in this section. This solution, developed by Garnier [9], generalizes those obtained for rectangular geometry. We extend it to the specific experimen-

TABLE III. Grid convergence for the 3D case with two meshes: $A$ is $80(r), 120(\theta), 20(z)$, and $B$ is $120(r)$, $180(\theta), 18(z)$. Around 50000 meshes are used in both case $A$ and $B$ to discretize the central zone under the heater.

\begin{tabular}{|c|c|c|c|c|c|c|c|c|c|}
\hline$T(\mathrm{~K})$ & Mesh & $\begin{array}{c}u(25 ; 2) \\
\mathrm{mm} \mathrm{s}^{-1}\end{array}$ & $\begin{array}{c}u(30 ; 2) \\
\mathrm{mm} \mathrm{s}^{-1}\end{array}$ & $\begin{array}{c}u(38 ; 2) \\
\mathrm{mm} \mathrm{s}^{-1}\end{array}$ & $\mathrm{Pe}_{\text {Max }}$ & $\mathrm{Pe}_{\text {average }}$ & $\begin{array}{l}\% \text { mesh } \\
\text { Pe } \leq 1\end{array}$ & $\begin{array}{l}\% \text { mesh } \\
\mathrm{Pe} \geq 10\end{array}$ & Flux (W) \\
\hline \multirow[t]{3}{*}{1} & $2 \mathrm{D}$ & 1.60 & 1.25 & 0.7 & & & & & 0.0674 \\
\hline & 3D $A$ & 1.55 & 1.2 & 0.68 & 23 & 1.9 & 48 & 2.9 & 0.0662 \\
\hline & $3 \mathrm{D} B$ & 1.55 & 1.15 & 0.65 & 21 & 1.39 & 48 & 1.17 & 0.0651 \\
\hline \multirow[t]{3}{*}{2.9} & $2 \mathrm{D}$ & 3 & 2.5 & 1.75 & & & & & 0.227 \\
\hline & 3D $A$ & 2.9 & 2.4 & 1.75 & 48 & 4.64 & 24 & 17 & 0.222 \\
\hline & 3D $B$ & 2.8 & 2.4 & 1.75 & 43 & 3.3 & 20.6 & 8.9 & 0.227 \\
\hline \multirow[t]{3}{*}{5} & $2 \mathrm{D}$ & 2.5 & 4 & 2.5 & & & & & 0.420 \\
\hline & 3D $A$ & 3.5 & 3.25 & 2.5 & 68 & 6.73 & 20 & 24 & 0.413 \\
\hline & 3D $B$ & 3.4 & 2.75 & 2.2 & 98 & 6.4 & 8.9 & 22 & 0.457 \\
\hline
\end{tabular}


TABLE IV. Adimensionnal scale.

Temperature

Radial length $(x)$

Height $(z)$

Velocity $(u, w)$

Pressure

Density

tal case of Favre which considers a cooled bottom boundary. The governing equations for the fluid layer are expressed in a cylindrical reference frame, but to approach the rectangular solution, a change of variable is done, $r=R_{1}+x$. The basic flow being axisymmetric, it implies $\frac{\partial}{\partial \theta} \equiv 0, v \equiv 0$. Henceforth, in all the equations the variables will appear in nondimensional form notified by a star superscript. The scalings are shown in Table IV. The method is based on the expansion of all unknown variables in terms of the aspect ratio considered as a small parameter $\varepsilon=h / L$, as follows:

$$
u^{*}\left(x^{*}, z^{*}\right)=\sum_{i=0}^{n} \varepsilon^{i} u_{i}^{*}\left(x^{*}, z^{*}\right)+O\left(\varepsilon^{n}\right) .
$$

The above expansion is introduced in the governing equations. The zeroth-order system can be solved analytically. The temperature solution exhibits a linear $z$ dependence because cooled bottom boundary $T_{0}^{*}\left(x^{*}, z^{*}\right)=\mu_{B} T_{\text {cond }}^{*}\left(x^{*}, z^{*}\right)$, where the nondimensional conducting profile of temperature $T_{\text {cond }}^{*}\left(x^{*}\right)$, is defined as

$$
T_{\text {cond }}^{*}\left(x^{*}\right)=1-\frac{\ln \left(1+\phi x^{*}\right)}{\ln (1+\phi)},
$$

$\phi=L / R_{1}$ characterizing the curvature of the cavity. We introduce $\Phi\left(x^{*}\right)$ as the horizontal temperature gradient of the conducting temperature profile,

$$
\Phi\left(x^{*}\right)=\frac{d T_{\text {cond }}^{*}}{d x^{*}}=-\frac{1}{\ln (1+\phi)} \frac{\phi}{1+\phi x^{*}} .
$$

The zeroth-order vertical velocity $v_{0}^{*}$ vanishes, while the horizontal component is

$$
\begin{aligned}
u_{0}^{*}\left(x^{*}, z^{*}\right)= & \mu_{B} \frac{\Phi\left(x^{*}\right)}{\operatorname{Pr}}\left(\frac{\operatorname{Ra}}{240}\left(10 z^{* 4}-27 z^{* 2}+14 z^{*}\right)\right. \\
& \left.-\frac{\operatorname{Ma}}{4}\left(3 z^{* 2}-2 z^{*}\right)\right) .
\end{aligned}
$$

In the case of a rectangular geometry, terms of order equal or greater than zero are all vanishing, so an exact solution for the basic state can be found. The curvature of the cavity implies a $x$ dependence of all variables which induces nonzero first-, second-, and higher-order terms. The system of first-order equations, thanks to the zeroth-order solution, can be solved in the same way. The first-order component of temperature is

$$
\begin{aligned}
T_{1}^{*}\left(x^{*}, z^{*}\right)= & \mu_{B}^{2} \frac{\Phi^{2}\left(x^{*}\right)}{240}\left(\frac { \mathrm { Ra } } { 4 2 0 } \left(100 z^{*_{7}}-567 z^{*_{5}}+490 z^{*_{4}}\right.\right. \\
& \left.+175 z^{*}-198 \mu_{B} z^{*}\right)-\operatorname{Ma}\left(9 z^{*_{5}}-10 z^{* 4}-5 z^{*}\right. \\
& \left.\left.+6 \mu_{B} z^{*}\right)\right)
\end{aligned}
$$

The expression of the first-order horizontal velocity component $u_{1}^{*}$ is determined by the equation

$$
\frac{\partial^{3} u_{1}^{*}}{\partial z^{* 3}}=\frac{\operatorname{Ra}}{\operatorname{Pr}} \frac{\partial T_{1}^{*}}{\partial x^{*}}+\frac{\partial}{\partial z}\left(u_{0}^{*} \frac{\partial u_{0}^{*}}{\partial x^{*}}\right) .
$$

Results for the basic flow. The theoretical results developed in the previous section are valid for a small aspect ratio, typically $\varepsilon \leq 0.05$. In the studied configuration where $L$ $=35 \mathrm{~mm}$, this condition required a height such that $h$ $\leq 1.75 \mathrm{~mm}$. We choose $h=1 \mathrm{~mm}(\varepsilon=0.04)$ in the present simulation, consequently the Bond number is fixed at $\mathrm{Bd}$ $=0.125$. For this set of parameters, a typical basic flow is obtained for $\Delta T=0.1 \mathrm{~K}(\mathrm{Ma}=7.34)$.

Analytical and numerical vertical temperature profiles are shown in Fig. 4 for three different radii. The linear dependence in $z$ of the temperature profile is clear. The first-order solution is more accurate but is not conclusive because of the strong variation in $z$ of the zeroth-order solution. Radial velocity profiles at three different radii are presented in Fig. 5. As the Bond number is much lower than 1, the thermocapillary effects prevail. The expression of $u_{0}$, Eq. (3), can be reduced only to the Marangoni term:

$$
u_{0}^{*}\left(x^{*}, z^{*}\right) \simeq-\mu_{B} \frac{\Phi\left(x^{*}\right)}{\operatorname{Pr}} \frac{\mathrm{Ma}}{4}\left(3 z^{* 2}-2 z^{*}\right) \text {. }
$$

This expression represents the well-known parabolic profile of a return flow with a $x$-dependent term $\Phi(x)$. For large values of $h$, comparison between experimental data due to Favre and numerical simulations are shown in Figs. 6 and 7. The conditions for both simulation and experiment are $h$ $=14.9 \mathrm{~mm}(\mathrm{Bd}=27.7), \Delta T=2.9 \mathrm{~K}(\mathrm{Ma}=47255)$, moreover, for the simulation, the free surface is assumed to be insulating $(\mathrm{Bi}=0)$. The analytical solution is less accurate in this case because the aspect ratio is too large $(\varepsilon>0.05)$.

Figure 6 gives the experimental and numerical radial velocity as a function of the depth $z$. The velocity profiles are no longer parabolic because the Bond number is high, and, furthermore, gravity plays a significant role. The agreement between the numerical and experimental results indicates that the assumptions of free surface, flat and adiabatic, are relevant for the resolution of the flow.

Figure 7 presents the experimental and numerical isotherms of the basic flow in the same configuration. In the lower zone, horizontal isotherms indicates that the bottom is cold. A thermal boundary layer develops along the cold vertical wall on the right. A difference with the numerical simulation is the behavior of the isotherms near the free surface. Some experimental isotherms hatch on the free surface which proves that it is not strictly insulating contrary to the assumptions made in the simulation. It would be necessary to take into account the thermal losses by convection over the 


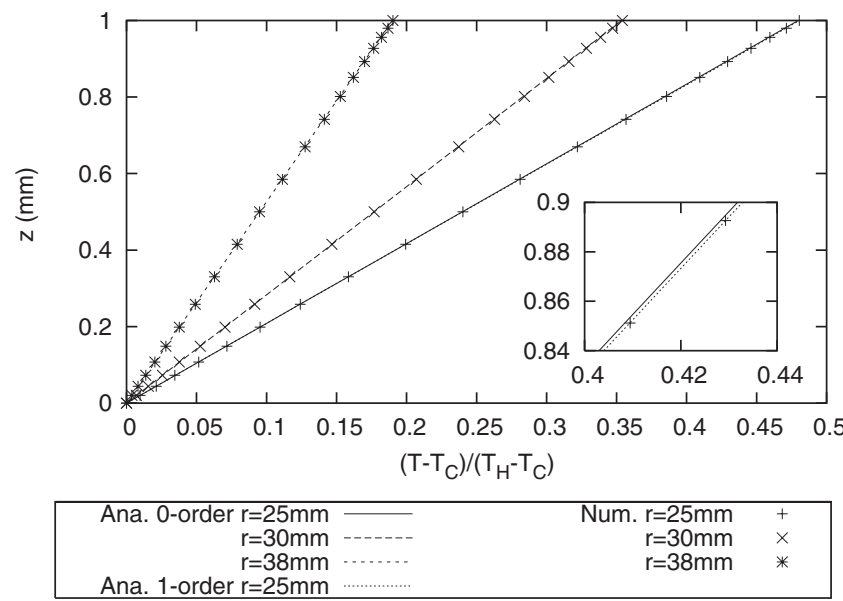

FIG. 4. Nondimensional axial temperature profiles at three different radii $(25,30$, and $38 \mathrm{~mm})$ and $\mathrm{Bi}=5 ; h=1 \mathrm{~mm}(\mathrm{Bd}=0.125)$, $\triangle T=0.1 \mathrm{~K}(\mathrm{Ma}=7.34)$.

free surface, the problem being to determine a value of the heat transfer coefficient (or Bi).

\section{B. Two-dimensional instabilities}

According to the stability diagram, the primary instability corresponds to small heights of fluid, $h \leq 3 \mathrm{~mm}$. The basic flow, which is a return flow, destabilizes and gives birth to rolls of a circular axis surrounding the heater. For approximately $\Delta T=1 \mathrm{~K}$, the first roll appears near the hot side as predicted by the theory [13] for fluids of Prandtl number $\operatorname{Pr} \geq 4$. Instability propagates then toward the cold edge with the appearance of new corotating rolls. The critical value of $\Delta T$ (or Ma) for which the first roll appears is hard to determine because the rolls seems to be a part of the basic flow. Moreover, experimental thresholds reported by Favre were found using a low accuracy shadowgraphic visualization method. So values reported on the stability diagram $(3.4 \mathrm{~K}$ in this case) are greater than the numerical ones because Favre could simply not visualize very small rolls. The number of

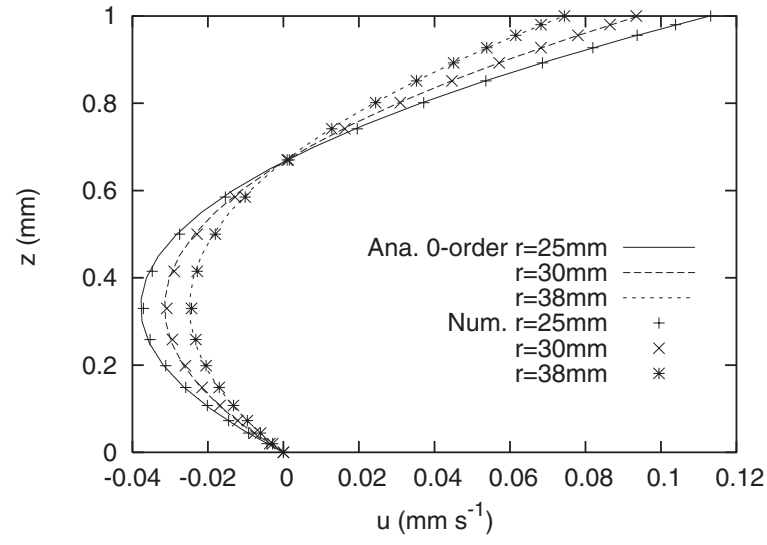

FIG. 5. Nondimensional radial velocity profiles at three different radii $(25,30$, and $38 \mathrm{~mm})$ and $\mathrm{Bi}=5 ; h=1 \mathrm{~mm}(\mathrm{Bd}=0.125) \triangle T$ $=1 \mathrm{~K}(\mathrm{Ma}=7.34)$.

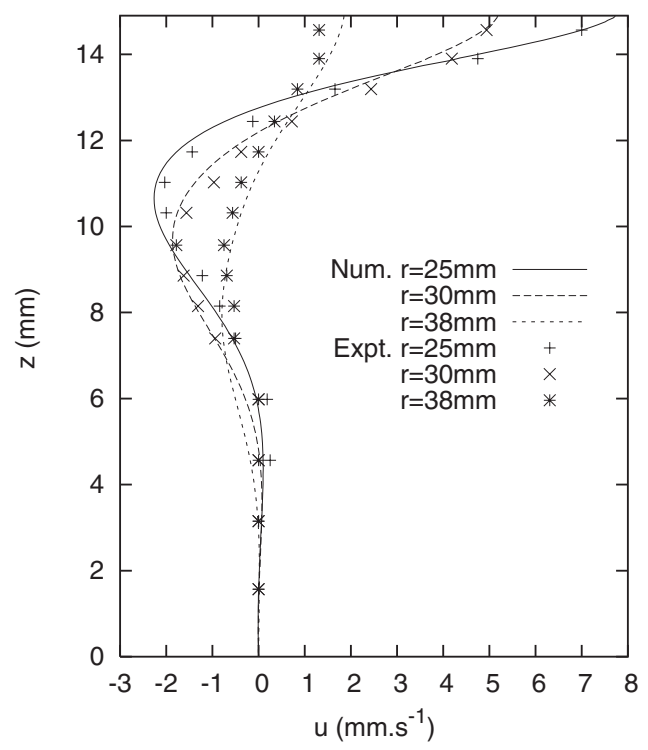

FIG. 6. Experimental and numerical radial velocity profiles at three different radii; $h=14.9 \mathrm{~mm}(\mathrm{Bd}=27.7), \Delta T=2.9 \mathrm{~K} \quad(\mathrm{Ma}$ $=47255$ ).

corotating rolls depends on the Bond number and the aspect ratio of the cavity. The numerical simulation indicates that the aspect ratio influences only the number of corotating rolls present in the cavity. On the contrary, the Bond number does not change the wavelength of the instability but there is a critical value $\left(\mathrm{Bd}_{c} \simeq 1\right)$ beyond which the corotating rolls could not exist whatever the value of the Marangoni number. This can be explained by the fact that the corotating roll is a thermocapillary instability, when $\mathrm{Bd}>1$, the thermocapillary forces being weaker than the thermogravitational force.

For the experimental conditions $h=2 \mathrm{~mm}(\mathrm{Bd}=0.5)$, when $\Delta T$ is increased above a certain secondary threshold $\Delta T_{c}$, bidimensional oscillating instabilities are observed in the computations. Instabilities grow starting from a basic
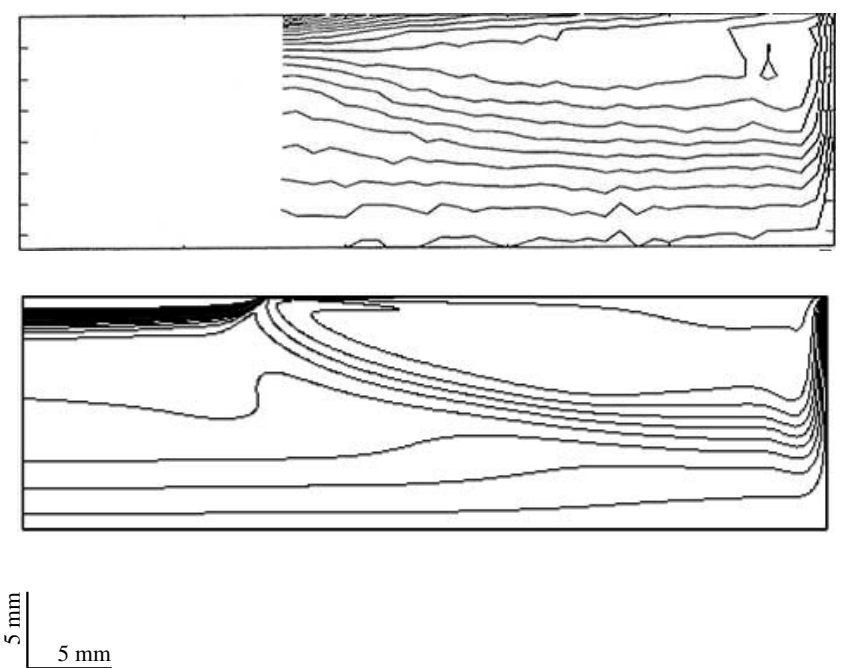

FIG. 7. Experimental (top) and numerical (bottom) isotherms of the basic flow for $h=14.9 \mathrm{~mm}, \triangle T=2.9 \mathrm{~K}(\mathrm{Ma}=47255)$ (20 isotherms between 0.04 and $1.54 \mathrm{~K}$ ). 


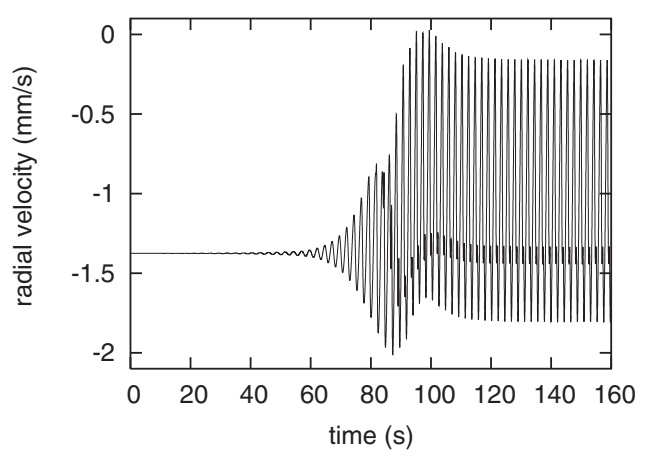

FIG. 8. Typical temporal evolution of the corotating instability.

flow obtained by a stationary resolution of the governing equations. The disturbance appears at its early stage as sinusoidal oscillations whose amplitude grows exponentially (see Fig. 8). It can be expressed for the temperature, for example, as

$$
T(r, \theta, z, t)=T(r, \theta, z) e^{\beta_{I} t} \cos \left(\beta_{I I} t\right),
$$

where $\beta_{I}$ is the growth rate of instability and $\beta_{I I}$ represents the oscillation frequency. The growth rate value of the instability is easily obtained in a semilogarithmic graph. After this growing phase, the instability stabilizes in nonsinusoidal oscillations with constant amplitude. Figure 9 shows temporal evolution of the radial velocity at a monitoring point $P(r$ $=30 \mathrm{~mm}$ and $z=1 \mathrm{~mm}$ ) over one period. The destabilization came from the first roll and consists in a periodic release of a new roll starting from the main one.

The new threshold, depending on the Bond number, could be determined precisely with a method due to Li et al. [24]. Plotting $\beta_{I}$ as a function of $\Delta T(\mathrm{Ma})$ allows us to determine, by linear regression, the critical value of the Marangoni number $\left(\mathrm{Ma}_{c}\right)$ for which instability occurs for $\beta_{I}=0$. In Fig. 10 , we can deduce a critical temperature difference of $9.4 \mathrm{~K}$ $\left(\mathrm{Ma}_{c}=2760\right)$. The value of $\Delta T$ corresponding to the appearance of hydrothermal waves in the stability diagram by Favre is $4 \mathrm{~K}(\mathrm{Ma}=1150)$, so Favre did not mention this temporal two-dimensional instability because hydrothermal waves appeared before, and experimentally these two-dimensional instabilities could not be observed. These two-dimensional oscillations were not the subject of a thorough study because Favre did not observe them. A lot of numerical publications already studied them in the past [14-16] because they do not require three-dimensional simulations. Experimentally some authors have reported them in a rectangular pool and they are often erased by three-dimensional instability.

\section{Three-dimensional instabilities: Comparison with experimental data}

\section{High height of fluid: Ray-type instability}

The "ray"-type instability has been simulated only in a fraction of $36^{\circ}$ of the cavity with $h=14.9 \mathrm{~mm}$. Indeed, due to the large height of fluid, we had to consider a vertical heater and periodic conditions on the lateral boundary to allow a

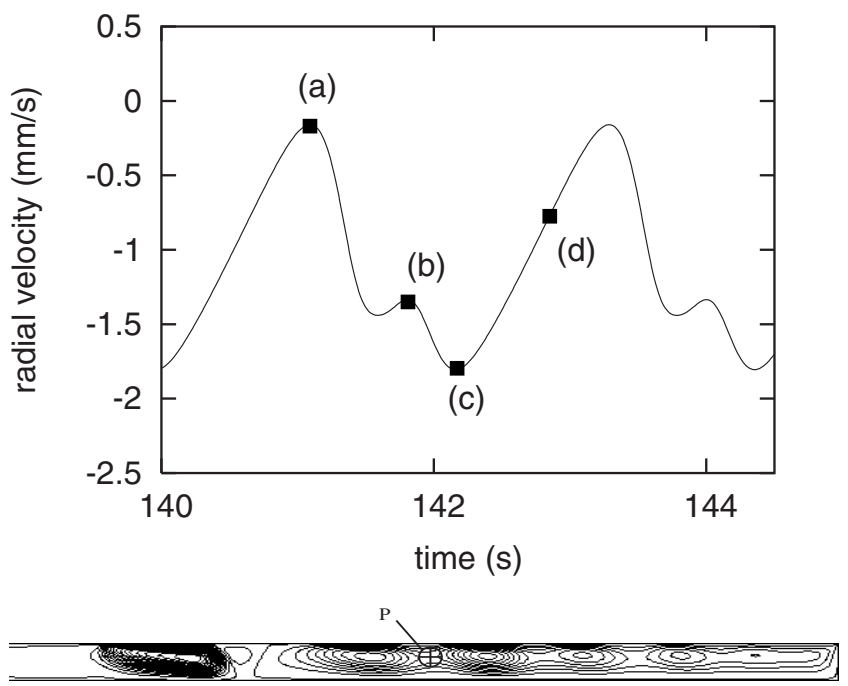

(a) $\mathrm{t}=140.9 \mathrm{~s}$

(b) $\mathrm{t}=141.5 \mathrm{~s}$

(c) $\mathrm{t}=142.1 \mathrm{~s}$

(d) $\mathrm{t}=142.7 \mathrm{~s}$

\begin{tabular}{l} 
唄 \\
\multicolumn{7}{|c}{$4 \mathrm{~mm}$} \\
\hline
\end{tabular}

FIG. 9. Contour of stream function $\Psi$ over one period for $\Delta T$ $=15 \mathrm{~K}(\mathrm{Ma}=4410)$ and $h=2 \mathrm{~mm}(\mathrm{Bd}=0.5)$. From top to bottom $\Psi_{\text {max }}$ is $\{1,9 ; 1,62 ; 1,48 ; 1,66\} 10^{-5} \mathrm{~kg} \mathrm{~s}^{-1}$.

fine mesh and reasonable computation times $[160(r)$ $\times 64(\theta) \times 60(z)]$. We have chosen to model $36^{\circ}$ of the cavity because the wave number predicted by Favre for this kind of instability was 10 . Moreover, computations were performed with a cavity with a maximal angle of $45^{\circ}$ but in this case the ray instability does not appear. For $\Delta T=5 \mathrm{~K}$, isotherms on the surface obtained with a stationary computation are shown in Fig. 11. In order to obtain a similar visualization of the rays, the azimuthal temperature gradient $(\partial T / \partial \theta)$ is computed. Moreover, an annular zone near the heater has been hatched because the shadow-graphics method was perturbed by the inner meniscus and the heater appears larger than it really is in the experimental visualization. A reconstitution to 


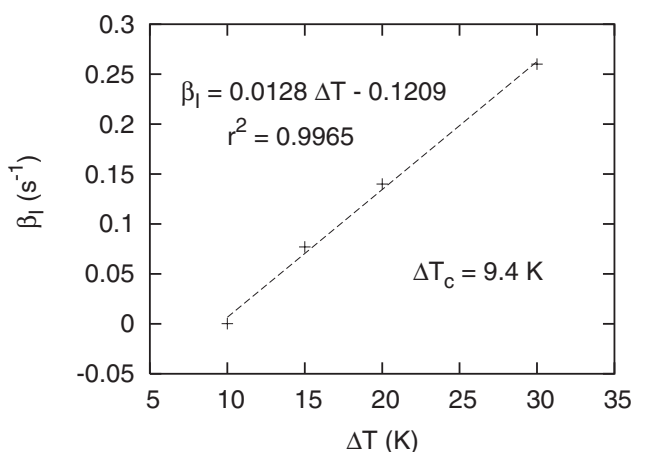

FIG. 10. Growing rate of the bidimensional instability $\left(\beta_{I}\right)$ for different applied thermal difference $\Delta T$.

$360^{\circ}$ of the azimuthal temperature gradient is compared to the shadow-graphic view obtained experimentally by Favre in Fig. 12. The unstationary secondary instability of the rays described by Favre corresponding to the oscillation of the structure around their initial position had not been simulated because it needed to simulate the full cavity.

\section{Intermediate height of fluid: Petal-type instability}

The petal instability has been recovered by a threedimensional simulation of a 4-mm-height layer $(\mathrm{Bd}=2)$ with a horizontal heater and a cooled bottom boundary. The mesh used for the simulation is $80(r) \times 120(\theta) \times 30(z)$ and 80000 more cells under the heater. For $\Delta T=10 \mathrm{~K}(\mathrm{Ma}=11744)$, a visualization of azimuthal temperature gradient $(\partial T / \partial \theta)$ is compared to the shadow-graphic view obtained experimentally by Favre in Fig. 13. The experimental visualization exhibits 14 structures against 16 for the numerical one. For this value of the Marangoni number, the numerical structures are stationary contrary to the prediction of the diagram of stability in which for $\Delta T \geq 6.5 \mathrm{~K}$ the petals rotate around the heater. The flow near the heater is quite axisymetric and

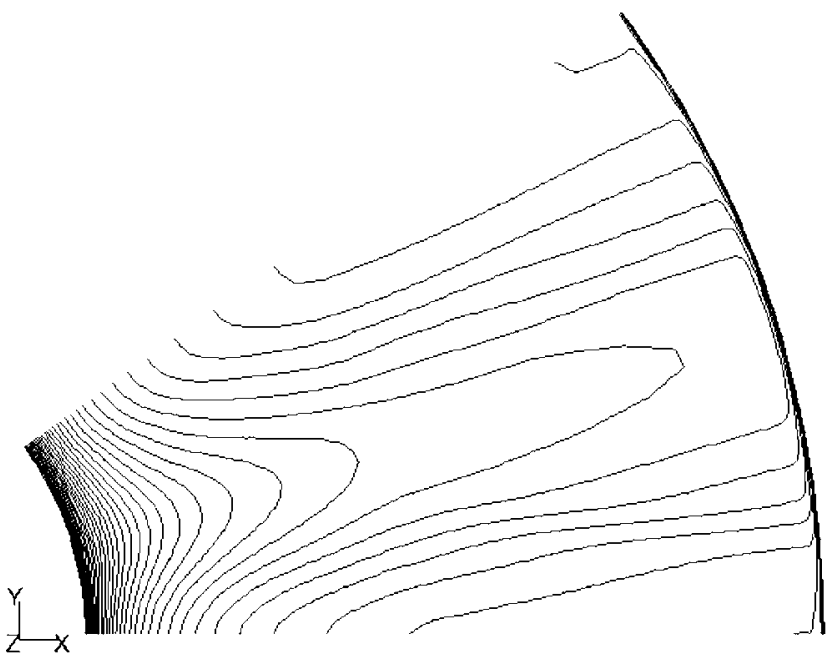

FIG. 11. Isotherms contours on the free surface for $\Delta T=5 \mathrm{~K}$ $(\mathrm{Ma}=81475)$ and $h=14.9 \mathrm{~mm}(\mathrm{Bd}=27.7)(80$ isovalues between 0 and $5 \mathrm{~K}$ ).
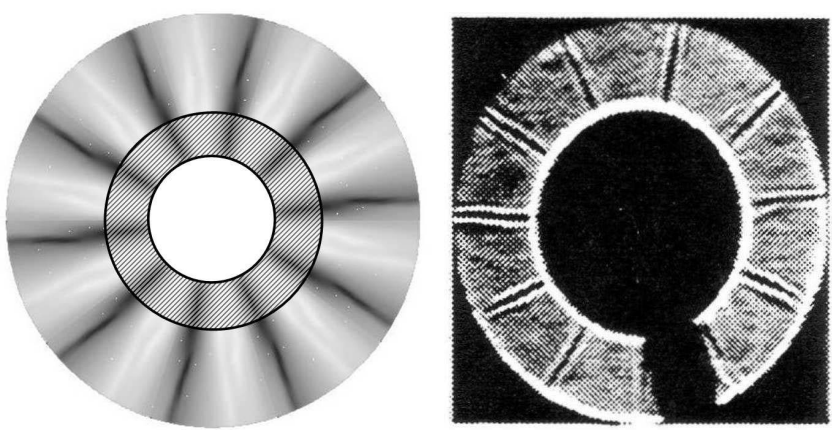

FIG. 12. Numerical $(\Delta T=5 \mathrm{~K}, \mathrm{Ma}=81475)$ and experimental $(\Delta T=10 \mathrm{~K}, \mathrm{Ma}=162950)$ visualization of the stationary ray-type instability for $h=14.9 \mathrm{~mm}(\mathrm{Bd}=27.7)$, the hatched zone corresponds to the zone hidden by the inner meniscus in the experimental visualization.

bidimensional, and instability occurs at $r=20 \mathrm{~mm}$, corresponding to the limit of the zone hidden by the inner meniscus. The fluid near the top surface moves faster periodically with $\theta$. This produces a horizontal return flow between theses structures. In fact, these petals are similar to non-fullydeveloped rays. For this intermediate height of fluid this instability fills all the height of fluid but is unable to reach the outer wall because the bottom is cooled.

\section{Small height of fluid}

Primary instability: The co-rotative roll instability (CR). The first observed instability is the corotative roll twodimensional instability already discussed in Sec. III B. The visualization of filled contours of the radial thermal gradient $(\partial T / \partial r)$ is similar to the experimental shadow-graphic visualization of this instability as shown in Fig. 14. As predicted by Favre, the two-dimensional (2D) oscillating instability has not been observed in the 3D simulation because hydrothermal waves appeared.

Secondary instability: Hydrothermal waves $(H W)$. The hydrothermal waves are more difficult to simulate because they need a full 3D simulation with a fine discretization along the $\theta$ direction. The hydrothermal waves appear in the same way as the bidimensional oscillations and stabilize after around $200 \mathrm{~s}$ of calculation. The same technique is used
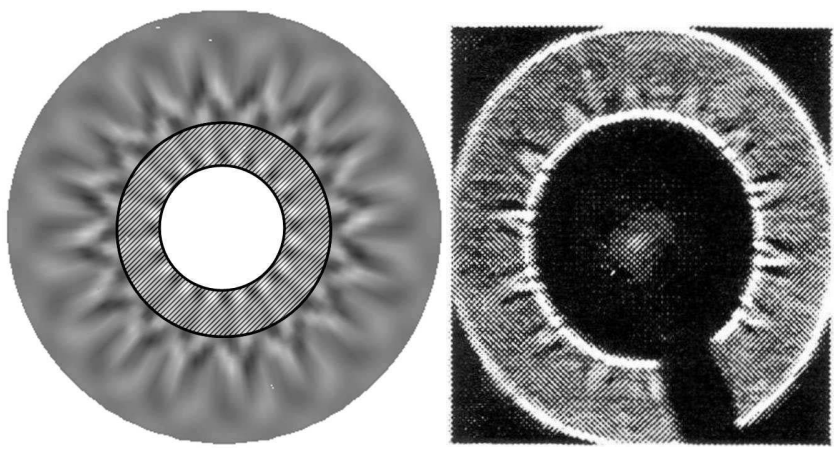

FIG. 13. Numerical $(\Delta T=10 \mathrm{~K}, \mathrm{Ma}=11744)$ and experimental $(\Delta T=6 \mathrm{~K}, \mathrm{Ma}=7047)$ visualization of the stationary petal instability for $h=4 \mathrm{~mm}(\mathrm{Bd}=2)$. 

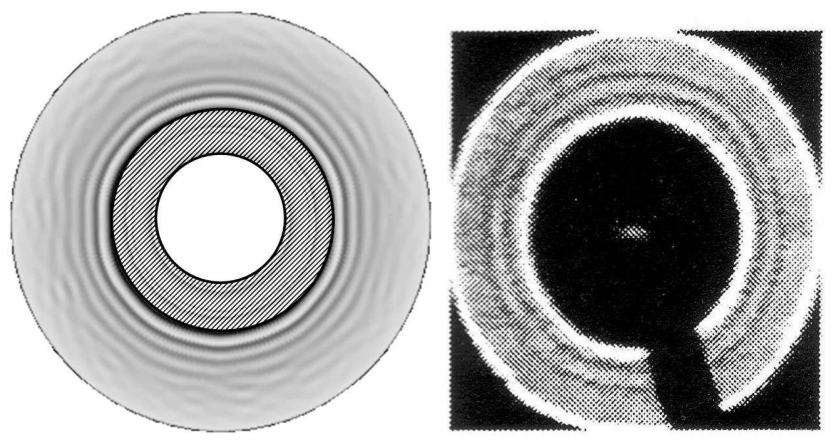

FIG. 14. Stationary corotative roll, experimental $\Delta T=3.2 \mathrm{~K}$, $(\mathrm{Ma}=761)$ and $h=1.8 \mathrm{~mm}(\mathrm{Bd}=0.41)$ shadow-graphic and numerical $\Delta T=7 \mathrm{~K}(\mathrm{Ma}=2055)$ and $h=2 \mathrm{~mm}(\mathrm{Bd}=0.5)$ visualization, scale between 0 and $100 \mathrm{~K} \mathrm{~m}^{-1}$.

to determine the critical Marangoni number value for which they appear. The initialization field of the computation is deduced from a stationary result with the same conditions which is normally an axisymetric flow.

In the experimental configuration using a $120(r)$ $\times 240(\theta) \times 15(z)$ plus 48990 meshes under the heater, hydrothermal waves were found for $h=1 \mathrm{~mm}(\mathrm{Bd}=0.127)$ and $\Delta T=5 \mathrm{~K}(\mathrm{Ma}=367)$. Figure 15 shows three-dimensional isosurfaces of azimuthal thermal gradient $(\partial T / \partial \theta)$. These surface waves look like curved branches formed in a wave source and disappearing in a wave sink. These waves propagate perpendicularly to the isotherm as in Favre et al. [2]. Two wave sources and two wave sinks were found. Hydrothermal waves obtained for other $\Delta T$ and different height were always with multiple sources and sinks.

Three simulations in this configuration for $\Delta T=3,5$ and $7 \mathrm{~K}$ allowed us to estimate the growth rate of this 3D hydrothermal wave as a function of $\Delta T$. The critical temperature difference for wave apparition is $\Delta T_{c}=3.8 \mathrm{~K} \quad(\mathrm{Ma}=279)$. From the diagram of stability of Favre et al. [2] for a liquid height equal to $1 \mathrm{~mm}$, the hydrothermal waves appear at the critical difference of temperature of $3 \mathrm{~K}(\mathrm{Ma}=220)$ in agree-

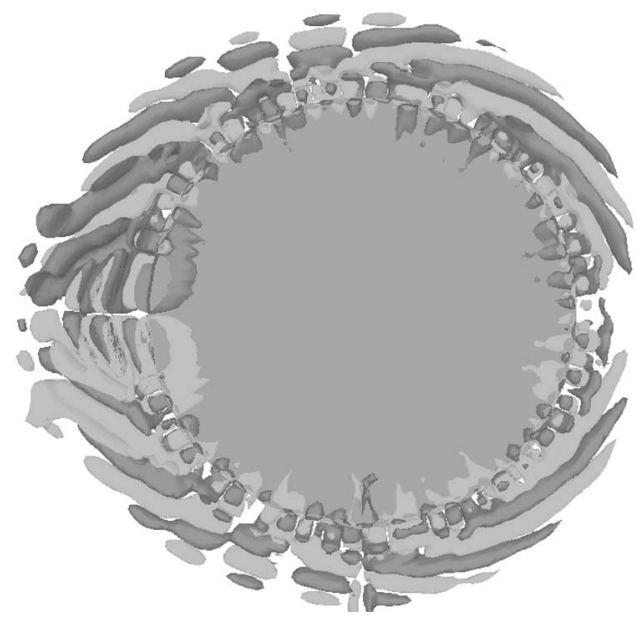

FIG. 15. Two 3D isosurface of azimuthal thermal gradient (7 $\mathrm{K} \mathrm{m}^{-1}$ in dark gray and $-7 \mathrm{~K} \mathrm{~m}^{-1}$ in light gray) for the case $h$ $=1 \mathrm{~mm}(\mathrm{Bd}=0.127)$ and $\Delta T=3 \mathrm{~K}(\mathrm{Ma}=220)$. ment with the simulations. Note that Shi and Imaishi [27] found a similar critical threshold for the apparition of hydrothermal waves $\Delta T_{c}=5.9 \mathrm{~K}$ for a configuration with $\operatorname{Pr}=6.7$, $h=1 \mathrm{~mm}, R_{1}=20 \mathrm{~mm}, R_{2}=40 \mathrm{~mm}$ with an adiabatic bottom and where the fluid is heated by the outer wall and cooled by the inner wall.

The spatiotemporal experimental and numerical diagrams of these hydrothermal waves are shown in Fig. 16. The numerical diagram was built with the fluctuating temperature $T^{\prime}$, defined by Eq. (2), and computed on a circle at $r$ $=20 \mathrm{~mm}$ on the free surface. The experimental diagram exhibits one wave source and one wave sink whereas the numerical diagram shows two sources and two sinks for the hydrothermal waves. The hydrothermal waves are a 3D extension of the corotating rolls as an azimuthally thermal "strength" where the $\Delta T$ increases.

Simulations of a higher layer of fluid corresponding to the experimental setup $(h=2 \mathrm{~mm})$ do not give proper results. In fact, the initial stationary computation does not give axisymetric results. Therefore the study of appearance of HWs were impossible. We found that this problem does not occur when the fluid is not cooled by the lower wall; in this configuration the radial extension of the instability is higher.

The main difference between simulation and experiment is the adiabatic free surface $(\mathrm{Bi}=0)$ which may change the number of branches but not the temperature threshold for the apparition of the waves.

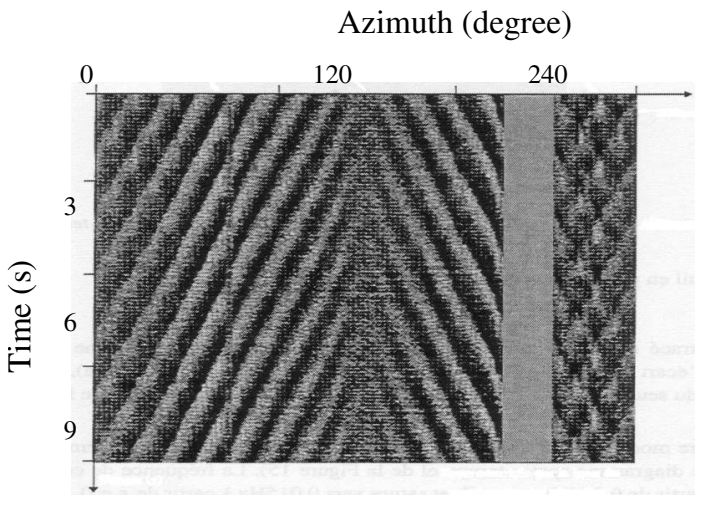

360

(a) 12 Hidden zone

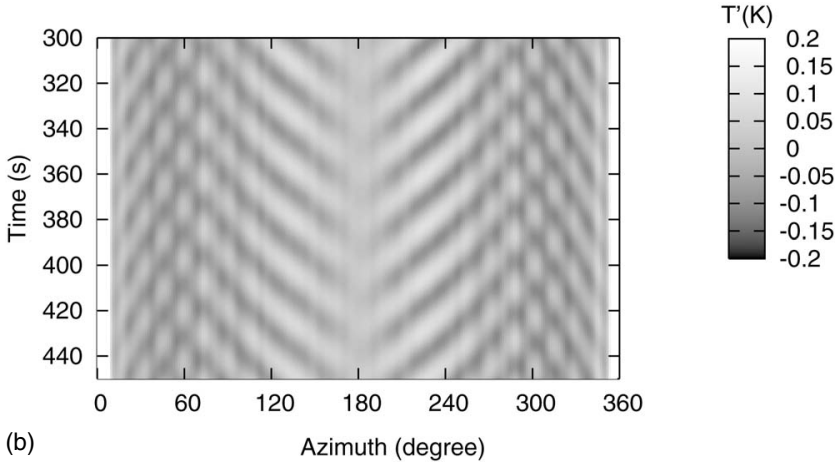

FIG. 16. Spatiotemporal diagrams: (a) experimental for $h$ $=2 \mathrm{~mm}(\mathrm{Bd}=0.51)$ and an unknown $\Delta T$, and (b) numerical for $h$ $=1 \mathrm{~mm}(\mathrm{Bd}=0.127)$ for $\Delta T=5 \mathrm{~K}(\mathrm{Ma}=367)$. 


\section{CONCLUSION}

A fine numerical simulation of thermoconvective instabilities of a fluid with an intermediate Prandtl number requires important resources. The buoyant-thermocapillary instabilities in a cavity filled with a fluid of Prandtl number of 10.3 heated on the surface in the center and cooled at the bottom and lateral boundary were simulated and compared with experimental results with success. The two-dimensional and three-dimensional simulations of the basic flow correspond to the experimental results of Favre [2] and of Castem 2000 simulations [3]. For the small aspect ratio of the cavity, the analytical solution of Garnier [9] was extended to the specific case of a cooled lower wall and was verified by the simulation. The different stationary instabilities observed by Favre have been studied and successfully simulated. The ray instability has been described for a high height of fluid. For an intermediate height of fluid petal instability has been simulated. We have shown that for small height of fluid the threshold of two-dimensional corotating instabilities is greater than that of the three-dimensional hydrothermal waves. It explains why experimentally no unstationary twodimensional instability was reported in this case by Favre [2]. Like in experimental observations, hydrothermal waves appear only when the dynamic Bond number is less than 1, i.e., when thermocapillary effects are predominant. Hydrothermal waves were more difficult to simulate, and we had to change the configuration of the heater to obtain valuable results. It can be explained by the fact that the inner and outer meniscus were not simulated. Moreover, the experimental heating boundary conditions are not strictly an imposed temperature.
[1] S. Schiller, U. Heisig, and S. Panzer, Electron Beam Technology (Wiley, Berlin, 1982).

[2] E. Favre, L. Blumenfeld, and F. Daviaud, Phys. Fluids 9, 1473 (1997).

[3] E. Favre, Ph.D. thesis, Institut National Polytechnique de Grenoble, 1997 (unpublished).

[4] S. Ostrach, Annu. Rev. Fluid Mech. 14, 313 (1982).

[5] R. Birikh, J. Appl. Mech. Tech. Phys. 7, 43 (1966).

[6] M. Smith and S. Davis, J. Fluid Mech. 132, 119 (1983).

[7] P. Laure, B. Roux, and H. Hadid, Phys. Fluids A 2, 516 (1990).

[8] J. Mercier and C. Normand, Phys. Fluids 8, 1433 (1996).

[9] N. Garnier, Ph.D. thesis, Université Paris, 2000 (unpublished).

[10] B.-C. Sim and A. Zebib, Int. J. Heat Mass Transfer 45, 4983 (2002).

[11] Y.-R. Li, N. Imashi, L. Peng, S.-Y. Wu, and T. Hibiya, J. Cryst. Growth 266, 88 (2004).

[12] D. Schwabe, U. Möller, J. Schneider, and A. Scharmmano, Phys. Fluids A 4, 2368 (1992).

[13] J. Mercier and C. Normand, Int. J. Heat Mass Transfer 45, 783 (2002).
[14] H. Hadid and B. Roux, J. Fluid Mech. 235, 1 (1992).

[15] D. Villers and J. Platten, J. Fluid Mech. 234, 487 (1992).

[16] J. Xu and A. Zebib, J. Fluid Mech. 364, 187 (1998).

[17] H. Hadid and B. Roux, J. Fluid Mech. 221, 77 (1990).

[18] A. Mancho and H. Herrero, Phys. Fluids 12, 1044 (2000).

[19] R. Riley and G. Neitzel, J. Fluid Mech. 359, 143 (1998).

[20] F. Daviaud and J.-M. Vince, Phys. Rev. E 48, 4432 (1993).

[21] J. Burguete and N. Mukolobwiez, Phys. Fluids 13, 2773 (2001).

[22] S. Hoyas, H. Herrero, and A. Mancho, Theor. Comput. Fluid Dyn. 18, 309 (2004).

[23] S. Hoyas, A. Mancho, H. Herrero, N. Garnier, and A. Chiffaudel, Phys. Fluids 17, 054104 (2005).

[24] Y.-R. Li, N. Imashi, T. Amazi, and T. Hibiya, J. Cryst. Growth 260, 28 (2004).

[25] Y.-R. Li, L. Peng, Y. Akiyama, and N. Imashi, J. Cryst. Growth 259, 374 (2003).

[26] Y.-R. Li, L. Peng, N. Imashi, and D. L. Zeng, Cryst. Res. Technol. 12, 1055 (2004).

[27] W. Shi and N. Imashi, J. Cryst. Growth 290, 280 (2006). 